\title{
ITERATED LITTLEWOOD-PALEY FUNCTIONS AND A MULTIPLIER THEOREM
}

\author{
W. R. MADYCH
}

ABSTRACT. A sufficient condition for a bounded function to be a multiplier of Fourier transforms on $L^{p}\left(R^{n}\right), 1<p<\infty$, is established. The classical case of Marcinkiewicz is properly included. The main tools used in obtaining this result are iterated variants of the classical LittlewoodPaley functions together with an $L^{p}$ estimate on certain maximal functions closely related to strong differentiability of multiple integrals.

Introduction. The classical version of the Marcinkiewicz multiplier theorem for Fourier integrals (also known as Mihlin's or Lizorkin's theorem) states that if $m(\xi)$ is a function on $R^{n}$ such that $\left|\xi^{a} D^{\alpha} m(\xi)\right|$ are bounded for all $\alpha$ 's in $\mathfrak{A}$, where $\mathfrak{A}$ is the collection of all multi-indexes whose components are zero or one, then the transformation $f \rightarrow M f$, defined on $C^{\infty}$ functions with compact support by $(M f)^{\wedge}(\xi)=m(\xi) \hat{f}(\xi)$, maps $L^{p}\left(R^{n}\right)$ boundedly into itself (see [4], [6]). The purpose of this note is to show that the theorem remains true if the set $\mathbb{A}$ is replaced by a more general collection of multi-indexes. The main tools used in obtaining our result are iterated variants of the classical Littlewood-Paley functions, $g$ and $g_{\lambda}^{*}$, together $w$ ith an estimate on certain maximal functions closely related to strong differentiability of multiple integrals.

We use the following notation: $R^{n}$ is a $n$-dimensional real Euclidean space whose elements are denoted by $x, z$ and $\xi$. Variables in $R^{1}$ are denoted by $y$ and $s$. $T^{n}$ represents the cone $\left\{t=\left(t_{1}, \cdots, t_{n}\right): t_{i}>0, i=1\right.$, $\cdots, n\}$ and $\Pi t=\Pi_{i=1}^{n} t_{i}$. For $x \in R^{n}$ and $t \in T^{n}, t x$ denotes the point $\left(t_{1} x_{1}, \cdots, t_{n} x_{n}\right)$ in $R^{n}$. If $\Omega$ is a measurable subset of $R^{n},|\Omega|$ denotes the Lebesgue measure of $\Omega$ and $t \Omega=\{t x: x \in \Omega\}$. For $x$ and $y$ in $R^{n}$, $\langle x, y\rangle=\sum_{i=1}^{n} x_{i} y_{i}$ and $|x|=\langle x, x\rangle^{1 / 2}$. All integrals are $w$ ith respect to Lebesgue measure, $d x$, and over all of $R^{n}$, unless denoted otherwise. $L^{p}=$ $L^{p}\left(R^{n}\right), 1 \leq p<\infty$, is the usual Banach space of measurable complex valued

Presented to the Society, January 18, 1974; received by the editors September $10,1973$.

AMS (MOS) subject classifications (1970). Primary 42A18, 44A25. 
functions $f$, such that $\|f\|_{p}=\left(\int|f(x)|^{p} d x\right)^{1 / p}<\infty ; L^{\infty}$ is the space of essentially bounded functions.

For $f$ in $L^{1}$, the Fourier transform, $f \rightarrow \hat{f}$, is defined by

$$
\hat{f}(\xi)=(2 \pi)^{-n / 2} \int f(x) e^{-i(x, \xi)} d x .
$$

The Fourier transform is defined on the space of tempered distributions in the the usual manner. All Fourier transforms and differentiations are to be interpreted in the distribution sense, unless they make sense otherwise. The convolution of two reasonable functions, $f_{1}$ and $f_{2}$, is defined by

$$
f_{1} * f_{2}(x)=\int f_{1}(x-z) f_{2}(z) d z,
$$

whenever this operation makes sense. If $f$ is in $L^{1}$ and $t \in T^{n}$ then $f_{t}$ is the function defined by $f_{t}(x)=\left(\Pi_{t}\right)^{-1} f\left(t^{-1} x\right)$, where $t^{-1}=\left(t^{-1}, \ldots, t_{n}^{-1}\right) \epsilon$ $T^{n}$.

Multi-indexes always have nonnegative integer components and are denoted by the symbols $\alpha, \beta$, and $\gamma$. If $x$ is in $R^{n}, x^{a}=\prod_{i=1}^{n} x_{i}^{a}$ and if $f$ is a distribution $D^{\alpha} f=\left(\partial / \partial x_{1}\right)^{\alpha} 1 \ldots\left(\partial / \partial x_{n}\right)^{a} n_{f} . \alpha \leq \beta$ means that $\alpha_{i} \leq \beta_{i}$, $i=1, \ldots, n$.

The symbol $C$ will be used generically for constants appearing in certain estimates. It need not be the same at different occurrences.

1. The Littlewood-Paley functions. A measurable complex valued function $\phi$ defined on $R^{1}$ is said to satisfy condition $A$ if there are positive constants $C$ and $\delta$ such that

(i) $|\phi(y)| \leq C(1+|y|)^{-1-\delta}$,

(ii) $\int_{-\infty}^{\infty} \phi(y) d y=0$, and

(iii) $\int_{-\infty}^{\infty}|\phi(s-y)-\phi(s)| d s \leq C|y|^{\delta}$.

Suppose that $k_{1}, \ldots, k_{n}$ satisfy condition $A$ and set $K(x)=\Pi_{i=1}^{n} k_{i}\left(x_{i}\right)$, $x=\left(x_{1}, \ldots, x_{n}\right)$. For $f$ in $L^{p}$ we define the Littlewood-Paley function $G_{K}(f)$ by the formula

$$
G_{K}(f, x)=\left\{\int_{T^{n}}\left|K_{t} * f(x)\right|^{2} \frac{d t}{\Pi t}\right\}^{1 / 2}
$$

Theorem 1. If $f$ is in $L^{p}, 1<p<\infty$, then $G_{K}(f)$ is in $L^{p}$ and $\left\|G_{K}(f)\right\|_{p}$ $\leq C\|f\|_{p}$ where $C$ is a constant which depends only on $K$ and $p$.

The theorem follows by iterating the vector-valued analogue of the 
Calderón-Zygmund inequality. To see this, write

$$
K_{t} * f(x)=\int_{-\infty}^{\infty} \frac{1}{t_{n}} k_{n}\left(\frac{z_{n}}{t_{n}}\right) \cdots\left(\int_{-\infty}^{\infty} \frac{1}{t_{1}} k_{1}\left(\frac{z_{1}}{t_{1}}\right) f(x-z) d z_{1}\right) \cdots d z_{n}
$$

and apply the results of $[1$, p. 359 and p. 363] $n$ times.

Theorem 2. Suppose $k_{1}, \cdots, k_{n}$ are even functions which satisfy condition $\mathrm{A}$ and set $K(x)=\Pi_{i=1}^{n} k_{i}\left(x_{i}\right)$. If none of the $k_{i}$ 's are identically zero, then for $f \in L^{p}, 1<p<\infty,\|f\|_{p} \leq C\left\|G_{K}(f)\right\|_{p}$ where $C$ is a constant depending only on $K$ and $p$.

The above fact is an immediate consequence of Plancherel's formula and Theorem 1. (Analogous calculations may be found in $[6$, p. 85] and $[5$, corollary to Theorem 7].)

Now suppose that $H$ is a nonnegative function in $L^{1}$ and $K$ satisfies the hypothesis of Theorem 1. If $f$ is in $L^{p}$, the Littlewood-Paley function $G_{K, H}(f)$ is defined by the formula

$$
G_{K, H}(f, x)=\left\{\int_{T^{n}} \int H_{t}(x-z)\left|K_{t} * f(z)\right|^{2} d z \frac{d t}{\Pi t}\right\}^{1 / 2}
$$

Observe that if $f$ is in $L^{2}$ then it follows from Plancherel's formula that $G_{K, H}(f)$ is in $L^{2}$ and $\left\|G_{K, H}(f)\right\|_{2} \leq C\|f\|_{2}$ where

$$
C=\left\{\|H\|_{1}\left\|\int_{T^{n}}\left|\hat{K}_{t}(\xi)\right|^{2} \frac{d t}{\Pi t}\right\|_{\infty}\right\}^{1 / 2} \text {. }
$$

Using arguments similar to those found in $[6, \mathrm{p} .91]$ and $[5$, Theorem 8], we see the following.

Theorem 3. Suppose the sublinear transformation $f \rightarrow(H f)^{*}$, defined by $(H f)^{*}(x)=\sup _{t \in T^{n}}\left|H_{t} * f(x)\right|$, maps $L^{q}$ boundedly into $L^{q}$ for each $q, 1<q$ $\leq \infty$. If $f$ is in $L^{p}, 2 \leq p<\infty$, then $G_{K, H}(f)$ is in $L^{p}$ and $\left\|G_{K, H}(f)\right\|_{p} \leq$ $C\|f\|_{p}$, where $C$ depends only on $K, H$, and $p$.

2. Tent functions. In order to apply the results of the previous section, we need a class of $H$ 's which satisfy the hypothesis of Theorem 3 . We begin by introducing an ordering in each $2^{n}$-ant of $R^{n}$.

Write $\operatorname{sgn} x=\left(\operatorname{sgn} x_{1}, \ldots, \operatorname{sgn} x_{n}\right)$. If $\operatorname{sgn} x=\operatorname{sgn} z$ and $\left|x_{i}\right| \leq\left|z_{i}\right|$, $i=1, \cdots, n$, we write $x \leq z$. 
Definition. $b$ is said to be a tent function if $b$ is a real valued nonnegative function in $L^{1}$ and $h(x) \geq h(z)$ whenever $\operatorname{sgn} x=\operatorname{sgn} z$ and $x \leq z$.

Theorem 4. Suppose $H$ is in $L^{1}$ and $|H(x)| \leq h(x)$, almost everywhere, where $b$ is a tent function. If $f$ is in $L^{p}, 1<p \leq \infty$, then $(H f)^{*}(x)=$ $\sup _{t \in T^{n}}\left|H_{t} * f(x)\right|$ is in $L^{p}$ and $\left\|(H f)^{*}\right\|_{p} \leq C\|f\|_{p}$ where $C$ depends only on $h, p$, and $n$.

The result is an immediate consequence of the following lemmas. For any locally inte grable $f$, define

$$
f^{*}(x)=\sup _{t \in T^{n}} \frac{1}{|t Q|} \int_{t Q}|f(x-z)| d z
$$

where $Q$ is the cube $\left\{x:-1 \leq\left|x_{i}\right| \leq 1\right\}$.

Lemma 1。(Jessen, Marcinkiewicz, and Zygmund). If $f \in L^{p}, 1<p \leq \infty$, then $f^{*}$ is in $L^{p}$ and $\left\|f^{*}\right\|_{p} \leq C\|f\|_{p}$ where $C$ is a constant which depends only on $n$ and $p$.

Lemma 2. Suppose $H$ satisfies the bypothesis of Theorem 4. If $f$ is in $L^{p}, 1<p \leq \infty$, then sup $\operatorname{st\in T}_{n}\left|H_{t} * f(x)\right| \leq 2^{3 n}\|b\|_{1} f^{*}(x)$.

Proof. The proof of Lemma 1 may be found in [3]. To see Lemma 2, we begin by estimating the $L^{1}$ norm of $b$ in terms of "lacunary parallelepipeds." Define $\bar{j}=\left(j_{1}, \ldots, j_{n}\right)$ where the $j_{i}$ 's are integers. $Q_{\bar{j}}$ denotes the parallelepiped $\left\{x: 2^{j_{i}<x}<\leq 2^{j_{i}+1}, i=1, \ldots, n\right\}$, and $Q_{\bar{j}}^{*}$ denotes the parallelepiped $\left\{x:\left|x_{i}\right| \leq 2^{j_{i}+1^{i}}, i=1, \ldots, n\right\}$. Clearly $Q_{\bar{j}} \subset Q_{\bar{j}}^{*}$ and $\left|Q_{\bar{j}}\right|=2^{|\bar{j}|}$, $\left|Q_{\bar{j}}^{*}\right|=2^{2 n_{2}|\bar{j}|}$, where $|\bar{j}|=j_{1}+\cdots+j_{n}$. Define $h_{1}$ by $h_{1}(x)=h(x)$ if sgn $x$ $>(0,0, \cdots, 0)$ and 0 otherwise. Observe that

$$
2^{-n} \sum_{\bar{j}} b\left(2^{\bar{j}}\right)\left|Q_{\bar{j}}\right| \leq \int h_{1}(x) d x \leq \sum_{\bar{j}} b\left(2^{\bar{j}}\right)\left|Q_{\bar{j}}\right|
$$

where $2^{\bar{j}}$ denotes the point $\left(2^{j} 1, \ldots, 2^{j} n\right)$ in $R^{n}$.

Now, if $f \in L^{p}, 1<p \leq \infty$, write

$$
\left|H_{t} * f(x)\right| \leq \int h_{t}(z)|f(x-z)| d z=\sum_{i=1}^{2^{n}} \int\left(h_{i}\right)_{t}(z)|f(x-z)| d z,
$$

where $h_{i}(x)=h(x)$ when $x$ is in the ith $2^{n}$-ant and zero otherwise, and observe that 


$$
\begin{aligned}
\int\left(h_{1}\right)_{t}(z)|f(x-z)| d z & =\sum_{\bar{j}} \int_{t}^{-1} z \in Q_{\bar{j}}(\Pi t)^{-1} h_{1}\left(t^{-1} z\right)|f(x-z)| d z \\
& \leq \sum_{\bar{j}} h_{1}\left(2^{\bar{j}}\right)\left|Q_{\bar{j}}^{*}\right| \frac{1}{\left|t Q_{\bar{j}}^{*}\right|} \int_{t Q_{\bar{j}}^{*}}|f(x-z)| d z .
\end{aligned}
$$

Hence

$$
\sup _{t \in T} \int\left(b_{1}\right)_{t}(z)|f(x-z)| d z \leq\left(\sum_{j} b_{1}\left(2^{j}\right)\left|Q_{j}^{*}\right|\right) f^{*}(x) \leq 2^{3 n}\left\|b_{1}\right\|_{1} f^{*}(x) .
$$

An analogous argument on $b_{2}, \cdots, b_{2}$, gives the desired result.

3. A multiplier theorem. Suppose $\mathscr{Q}$ is a set of multi-indexes. We say that $\mathfrak{Q}$ is nice if

(i) $\left(\Sigma_{\alpha \in Q}\left|x^{a}\right|\right)^{-2}$ is in $L^{1}$, and

(ii) if $\alpha \in \mathbb{Q}$ then any $\beta$ satisfying $\beta \leq \alpha$ is also in $\mathfrak{A}$.

The following are examples of nice sets of multi-indexes: the set mentioned in the introduction; $\mathbb{Q}=\{\alpha:|\alpha| \leq \kappa\}$, where $\kappa$ is any integer greater than $n / 2 ; \mathbb{A}=$ $\left\{\alpha: \alpha \leq \beta^{(i)}\right.$ for some $\left.i, i=1, \ldots, n\right\}$, where $\beta^{(i)}$ is the multi-index which contains $b_{i}$ in the $i$ th slot, zero elsewhere, and $\sum_{i=1}^{n}\left(b_{i}\right)^{-1}<2$.

For $t \in T^{n}$, we define $Q_{t}$ to be the set $\left\{x: t_{i}<\left|x_{i}\right|<2 t_{i}, i=1, \cdots, n\right\}$; $Q_{t}$ is the union of $2^{n}$ paralle lepipeds.

Theorem 5. Suppose $m$ is in $L^{\infty}$ and $\Sigma_{\alpha \in \mathbb{Q}}\left(1 /\left|Q_{t}\right|\right) \int_{Q_{t}}\left|\xi^{\alpha} D^{a} m(\xi)\right|^{2} d \xi$ $\leq B^{2}$ for all $t \in T^{n}$, where $\mathbb{Q}$ is a nice set of multi-indexes. Define $M f$ for $\bar{f}$ in $L^{2} \cap L^{p}$ by the formula $(M f)^{\wedge}(\xi)=m(\xi) \hat{f}(\xi)$. Then $\|M f\|_{p} \leq C B\left\|_{f}\right\|_{p}$ for $1<p<\infty$, where $C$ is independent of $f$.

The proof of the theorem consists of picking appropriate $K_{1}, K_{2}$ and $H$, establishing the inequality $G_{K_{1}}(M f, x) \leq C G_{K_{2}, H}(f, x)$, and applying Theorems 2 and 3.

Let $\phi$ be an even function in $C^{\infty}\left(R^{1}\right)$, which is not identically zero and whose support is contained in $\{y: 1 \leq|y| \leq 2\}$. Define $k_{1}$ and $k_{2}$ to be the inverse Fourier transforms of $\phi(y)$ and $[\phi(y)]^{2}$ respectively (as functions in $\left.R^{1}\right)$ and set $K_{1}(x)=\Pi_{i=1}^{n} k_{1}\left(x_{i}\right)$ and $K_{2}(x)=\Pi_{i=1}^{n} k_{2}\left(x_{i}\right)$. Let $H(x)=\left(\Sigma_{a \in \mathbb{Q}}\left|x^{a}\right|\right)^{-2}$, where $\mathbb{Q}$ is the nice set of multi-indexes of Theorem 5 . Clearly $K_{1}$ and $K_{2}$ satisfy the hypothes is of Theorems 1 and 2 and $H$ is a tent function. 
Lemma 3. If $m$ satisfies the hypothesis of Theorem 5 and $K_{1}, K_{2}, H$, and $M f$ are defined as above, then $G_{K_{2}}(M f, x) \leq C B G_{K_{1}, H}(f, x)$ where $C$ depends only on $K_{1}$ and $\mathfrak{Q}$.

Proof. Write

$$
\left(K_{2}\right)_{t} * M f(x)=\int K_{1}(z ; m, t)\left(K_{1}\right)_{t} * f(x-z) d z
$$

where

$$
K_{1}(z ; m, t)=(2 \pi)^{-n / 2} \int m(\xi) \Phi(t \xi) e^{i(z, \xi)} d \xi,
$$

and $\Phi(t \xi)=\Pi_{j=1}^{n} \phi\left(t_{j} \xi_{j}\right)$. Now

$$
\left|\left(K_{2}\right)_{t} * M f(x)\right|^{2} \leq I_{1} I_{2}
$$

where

$$
I_{1}=\int\left|\sum_{a \in \mathbb{Q}}\left(t^{-1} z\right)^{a} K_{1}(z ; m, t)\right|^{2} d z
$$

and

$$
I_{2}=\int(\Pi t) H_{t}(z)\left|\left(K_{1}\right)_{t} * f(x-z)\right|^{2} d z
$$

To estimate $I_{1}$, apply Plancherel's formula

$$
\int\left|\left(t^{-1} z\right)^{\alpha} K_{1}(z ; m, t)\right|^{2} d z=\int\left|\left(t^{-1}\right)^{\alpha} D^{\alpha}(m(\xi) \Phi(t \xi))\right|^{2} d \xi,
$$

and observe that

$$
\left(t^{-1}\right)^{\alpha} D^{\alpha}(m(\xi) \Phi(t \xi))=\sum_{\beta+\gamma=\alpha} C_{\beta}\left(t^{-1}\right)^{\beta}\left(D^{\beta} m(\xi)\right) D^{\gamma} \Phi(t \xi) .
$$

Since $\Phi(t \xi)$ is supported in $Q_{1 / t}=\left\{\xi: t_{i}^{-1} \leq\left|\xi_{i}\right| \leq 2 t_{i}^{-1}, i=1, \ldots, n\right\}$, the last two formulas imply that

$$
I_{1} \leq C \sum_{a \in Q} \int_{Q_{1 / t}}\left|\xi^{a} D^{a} m(\xi)\right|^{2} d \xi \leq C B^{2}\left|Q_{1 / t}\right| \cdot
$$

Substituting (2) and the formula for $I_{2}$ into (1) and integrating over $T^{n}$ gives us

$$
\int_{T^{n}}\left|\left(K_{2}\right)_{t} * M f(x)\right|^{2} \frac{d t}{\Pi t} \leq C B^{2} \int_{T^{n}} \int H_{t}(z)\left|\left(K_{1}\right)_{t} * f(x-z)\right|^{2} d z \frac{d t}{\Pi t},
$$

which is the desired result. 
Thus the theorem holds for $2 \leq p<\infty$. The fact that it remains true in the range $1<p \leq 2$, follows by duality (see [2, p. 100]).

\section{REFERENCES}

1. A. Benedek, A. P. Calderón and R. Panzone, Convolution operators on Banach space valued functions, Proc. Nat. Acad. Sci. U.S.A. 48 (1962), 356-365. MR 24 \#A3479.

2. L. Hörmander, Estimates for translation invariant operators in $L^{p}$ spaces, Acta. Math. 104 (1960), 93-140. MR 22 \#12389.

3. B. Jessen, J. Marcinkiewicz and A. Zygmund, Note on the differentiability of multiple integrals, Fund. Math. 25 (1936), 217-234.

4. W. Littman, C. McCarthy and N. Rivière, $L^{p}$-multiplier theorems, Studia Math. 30 (1968), 193-217. MR 37 \#6681.

5. W. R. Madych, On Littlewood-Paley functions, Studia Math. (to appear).

6. E. M. Stein, Singular integrals and differentiability properties of functions, Princeton Math. Series, no. 30, Princeton Univ. Press, Princeton, N. J., 1970. MR $44 \# 7280$.

DEPARTMENT OF MATHEMATICS, TEXAS A \& M UNIVERSITY, COLLEGE STATION, TEXAS 77843 